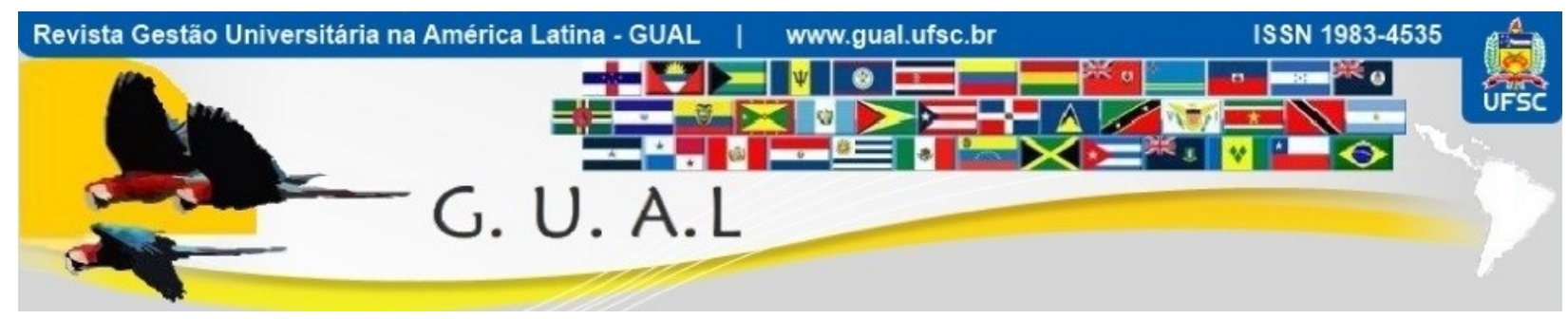

DOI: http://dx.doi.org/10.5007/1983-4535.2018v11n2p70

\title{
ESTUDO DOS FATORES ASSOCIATIVOS DOS ESTILOS DE APRENDIZAGEM DOS ACADÉMICOS DO CURSO DE CIÊNCIAS CONTÁBEIS
}

\section{ANALYSIS OF THE ASSOCIATIVE FACTORS RELATED TO THE LEARNING STYLES OF ACCOUNTING STUDENTS}

Cleston Alexandre dos Santos, Doutorando Universidade Federal de Mato Grosso do Sul - UFMS cleston.alexandre@ufms.br

Laurindo Panucci Filho, Doutorando Universidade Estadual do Norte do Paraná - UENP laurindopanucci@hotmail.com

Nelson Hein, Pós-Doutor Universidade Regional de Blumenau - FURB hein@furb.br

Recebido em 11/maio/2016

Aprovado em 20/março/2018

Sistema de Avaliação: Double Blind Review

Esta obra está sob uma Licença Creative Commons Atribuição-Uso. 


\title{
ESTUDO DOS FATORES ASSOCIATIVOS DOS ESTILOS DE APRENDIZAGEM DOS ACADÊMICOS \\ DO CURSO DE CIÊNCIAS CONTÁBEIS \\ DOI: http://dx.doi.org/10.5007/1983-4535.2018v11n2p70
}

\section{RESUMO}

Os estilos de aprendizagem detém abordagens inerentes às características de cada pessoa, o que torna necessário que cada docente desenvolva um planejamento de ensino que o possibilite trabalhar e interagir de forma dinâmica com os diferentes estilos de aprendizagem de seus acadêmicos. O presente trabalho tem como objetivo evidenciar os fatores associativos dos estilos de aprendizagem dos acadêmicos do curso de ciências contábeis da FURB. No tratamento metodológico, classifica-se como estudo descritivo, formal, ex post facto, em condições de campo, transversal, de rotina real, estatístico, utilizando-se de coleta de dados por meio de questionário. O estudo foi desenvolvido nos meses de maio e junho de 2015 e constituiu-se de 151 acadêmicos dos 271 matriculados e com frequência no curso. Os resultados revelam que predominou o estilo de aprendizagem convergente entre os alunos pesquisados. Por meio da análise de correspondência (ANACOR), com a utilização do software LHStat $\AA$, o mapa perceptual revelou a associação dos estilos de aprendizagem acomodador e divergente com os acadêmicos da fase inicial do curso, com o gênero feminino, com acadêmicos de idade até 20 anos e com os acadêmicos que tem uma alta dedicação de horas aos estudos por semana. $\mathrm{O}$ estudo apontou também a associação dos estilos assimilador e convergente com os acadêmicos da fase final do curso, do gênero masculino, com idade entre 21 a 25 anos e que também tem uma carga horária relevante de horas de dedicação aos estudos por semana.

Palavras-chave: Estilos de Aprendizagem. Inventário de David Kolb. Fatores Associativos. Análise de Correspondência. Acadêmicos de Ciências Contábeis.

\begin{abstract}
Learning styles hold approaches related to each person's characteristics. In the educational field, it is necessary that each teacher develop a teaching plan that allows him to work and interact in a dynamic way with the different learning styles of his students. The present study aims at demonstrating the associative factors related to the learning styles of students enrolled in the Accounting course at the Regional University of Blumenau (FURB), Brazil. As regards the method, this is a descriptive, statistical, cross-sectional study. It can be considered formal, ex post facto and field survey. Data was collected by means of a questionnaire and data collection took place in the months of May and June/2015. As participants, 151 of the 271 enrolled in the course took part in the study. Results revealed that the converging learning style prevailed among the participants. With the aid of correspondence analysis conducted with the software LHStat $\AA$, the perceptual map suggested the association of the accommodating and diverging learning styles with the students in the beginning of the course, with the female gender, with students of up to 20 years old and with the students who dedicate many hours of their week to study. As well, results indicated the association of the assimilating and converging learning styles with the students in the final stage of the course, with the male gender, with ages between 21 to 25 years old and with the ones who have a relevant number of hours available to study a week.
\end{abstract}

Keywords: Learning Styles. David Kolb's Inventory. Associative Factors. Correspondence Analysis. Accounting Students. 


\section{INTRODUÇÃO}

$\mathrm{O}$ estilo de aprendizagem de cada pessoa ou de cada aluno tem relação com o procedimento de ensino e a maneira de aprendizagem, do docente com o seu aluno no cumprimento do conteúdo programático das disciplinas (CERQUEIRA, 2000). Dessa forma, torna-se de grande valia o conhecimento do estilo de aprendizagem do acadêmico e a técnica didática que o docente utiliza com os alunos em atividades que envolvem situações teóricas e práticas, pois cada pessoa tende a apresentar estilos diferentes de aprendizagem.

Há argumentos que o estilo de aprendizagem evidencia como o indivíduo absorve e processa a informação recebida, e por esse raciocínio, pode ser observado de forma entrelaçada, como também interdependente na evolução das características específicas de cada acadêmico (KOLB, 1976). Com a identificação do estilo de aprendizagem do aluno e ao transmitir uma informação, o docente encontra e emprega a forma mais eficiente e eficaz de ensino.

O estilo de aprendizagem é caracterizado como sendo um método que um indivíduo tem para obter o conhecimento, cujo conhecimento é adquirido pelo modo específico e pessoal de cada um (KOLB, 1976). A literatura aponta que o estilo de aprendizagem não é o que a aluno aprende e sim a maneira como se comporta no processo do aprendizado. Portanto, esse comportamento pessoal contribui para a explicação do motivo de uma pessoa aprender todo alfabeto após a leitura de um livro de alfabetização, enquanto em situação diferente, outros podem obter o mesmo conhecimento por meio de brincadeiras com blocos de construção que contemple letras, por exemplo (REIS; PATON; NOGUEIRA, 2012).

A literatura mostra que o amplo conhecimento e entendimento dos variados estilos de aprendizagem possibilitam a melhoria da qualidade do processo de ensino-aprendizagem, já que a obtenção dessas informações impulsionam o desenvolvimento de métodos e técnicas de ensino de acordo com as características pessoais dos acadêmicos (CORDEIRO; DA SILVA, 2012). Dessa forma, identificar e entender o estilo de aprendizagem do aluno e os fatores associativos devem contemplar uma necessidade e preocupação contínua dos docentes e equipes pedagógicas das Instituições de Ensino (NOGUEIRA et al., 2013).

Segundo Lima Filho, Bezerra e Silva (2016) a identificação do estilo de aprendizagem do aluno oportuniza o docente a desenvolver mecanismos cognitivos que contribuem para a absorção das informações em paralelo ao direcionamento da sua metodologia tomando como base o estilo predominante e o estímulo dos estilos secundários. 
O fato de buscar conhecer e compreender o tipo do estilo de aprendizagem dos acadêmicos e os fatores que estão associados, torna-se fundamental para análise e revisão das metodologias utilizadas pelos docentes durante as atividades em sala de aula, o que possibilita mais compreensão sobre o que é pensado pelos alunos. Com base no exposto, foi elaborada a seguinte questão de pesquisa: quais são os fatores associativos dos estilos de aprendizagem dos acadêmicos do curso de ciências contábeis da Universidade Regional de Blumenau (FURB), Brasil? Deste modo, o objetivo é evidenciar os fatores associativos dos estilos de aprendizagem dos acadêmicos do curso de ciências contábeis da FURB.

Pesquisas têm sido aplicadas para estudar os estilos de aprendizagem por meio das várias teorias, o que possibilita uma melhor compreensão das características pessoais, como também auxilia docente no planejamento das suas atividades para um melhor entendimento do perfil dos alunos (TIRADOS, 1985; CERQUEIRA, 2000; REIS et al., 2012; NOGUEIRA; ESPEJO; REIS; VOESE, 2012; LIMA FILHO; BEZERRA; SILVA, 2016), e ainda, cria um espaço para novas pesquisas e com novas evidenciações e associações. Lima Filho, Bezerra e Silva (2016) argumentam que durante o processo de ensino-aprendizagem é que surge a possibilidade de promover a autonomia do discente no processo de construção do conhecimento, como também na capacidade de aprender.

O presente trabalho está estruturado em quatro partes sendo está a primeira, a segunda o referencial teórico, na terceira parte têm-se os procedimentos metodológicos, em seguida na quarta parte a análise e interpretação dos dados, e por fim, as considerações finais e recomendações.

\section{REFERENCIAL TEÓRICO}

Neste tópico será discorrido sobre Estilo de Aprendizagem de David Kolb, e em seguida, Abordagens de estudos anteriores.

\subsection{ESTILO DE APRENDIZAGEM SEGUNDO DAVID A. KOLB}

O estilo de aprendizagem compreende os meios, os métodos, as formas, o processo, como também o trajeto que um indivíduo faz uso para obter o conhecimento, ou seja, cada um tem sua forma de percepção, caminho e a maneira de aprender REIS et al. (2012, p.54) apontam que, conceitualmente, “o estilo de aprendizagem não é o que o aluno aprende e sim o modo como ele se comporta no aprendizado, sendo a forma como cada acadêmico se 
concentra, processa, internaliza e retêm nova e complexa informação". De acordo com Lima Filho, Bezerra e Silva (2016, p.97), “os alunos têm diferentes maneiras de aprender e manipulam as informações por métodos distintos e assim, fatores como personalidade, característica, níveis de dificuldade das tarefas e da aprendizagem estão fortemente envolvidos".

Kolb e Fry (1975) apresentaram um modelo composto de quatro elementos: a experiência concreta (EC); a observação reflexiva (OR); a formação de conceitos abstratos (CA); a experiência ativa (EA). Esse modelo pode ser representado pelo círculo de aprendizagem experimental, conforme Figura 1:

Figura 1 Círculo de aprendizagem experimental de Kolb

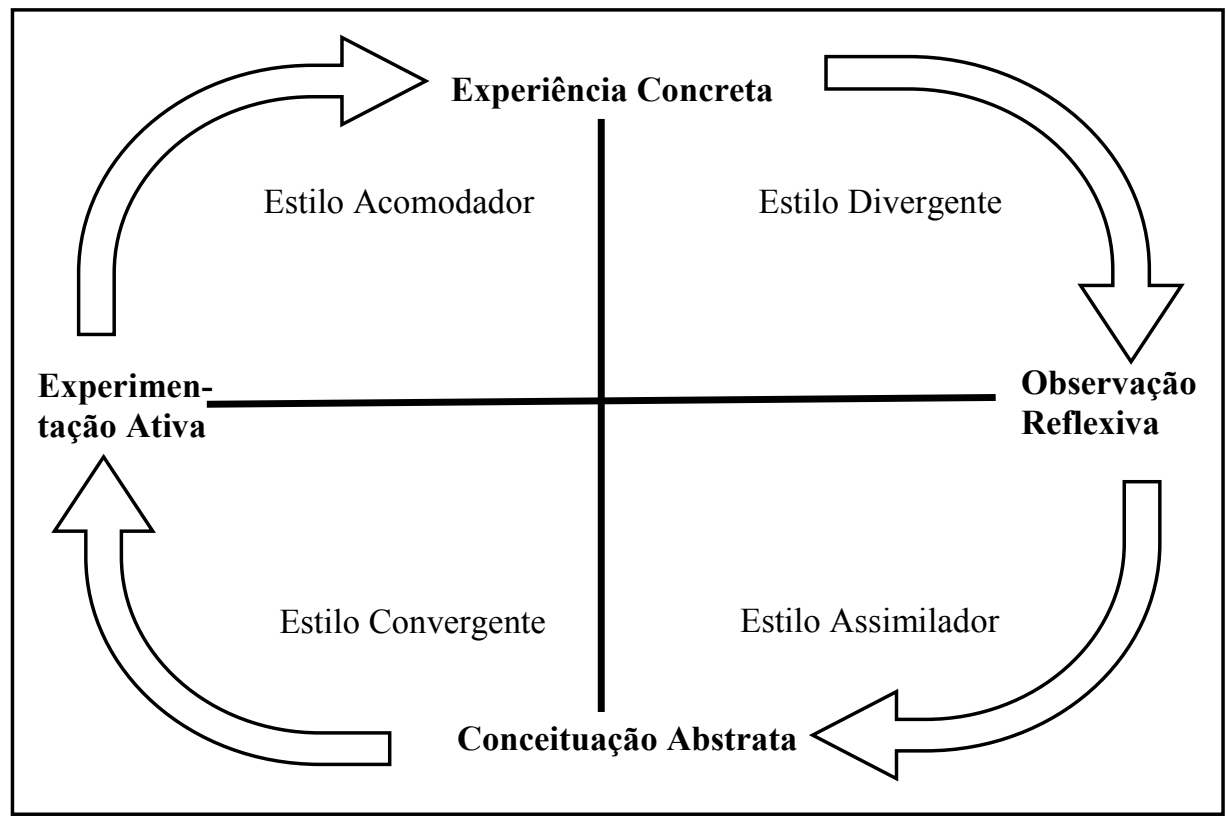

Fonte: adaptado de Kolb (1984).

Kolb e Fry (1975) destacam que o círculo de aprendizagem experimental pode ter início em um dos quatros quadrantes e que deve ser compreendido como uma espiral contínua. Os autores argumentam que o processo de aprendizagem tem início com um indivíduo levando a cabo uma ação particular e que veja o efeito da mesma (experiência concreta (EC)). Na seqüência, é preciso entender os efeitos da situação específica, de maneira como se essa ação fosse realizada e nas mesmas circunstancias, fosse possível adiantar o que seguiria a tomada da ação (observação reflexiva (OR)). No próximo quadrante, o propósito é o pensamento baseado no raciocínio lógico, em que os aprendizes tendem a ser mais 
orientados a símbolos e coisas do que a pessoas, o que faz assimilar melhor o aprendizado por meio do pensamento do que de descobertas como exercícios e simulações. (conceito abstrato (CA)). Por fim, tem pessoas que não gostam de situações de aprendizado passivo como assistir às aulas, e tendem a serem extrovertidos e tem disposição forte em realizar atividades práticas. (experimentação ativa (EA)).

Kolb e Fry (1975) apontam que a aprendizagem efetiva necessita o pleno domínio das quatro habilidades diferentes apresentadas acima: habilidades de experiência concreta (EC), de observação reflexiva (OR), de conceituação abstrata (CA), e por fim, de experimentação ativas (EA). Como há poucas pessoas que tem condições de somar todos os componentes do modelo, os autores recomendam a concentração de dois elementos para identificação dos estilos de aprendizagem.

Com base no círculo de aprendizagem e nas características de cada aluno, Kolb (1975) identificou quatro grupos de estudantes: os acomodadores, assimiladores, convergentes e divergentes, como pode ser evidenciado na Figura 1 e a seguir:

Estilo Acomodador: combina as etapas da Experiência Concreta (EC) e da Experimentação Ativa (EA). Nesse estilo os acadêmicos adaptam-se bem às circunstâncias imediatas e aprendem, ao fazer atividades e aceitando desafios; atuam mais pelo que sentem do que pela análise lógica; são considerados intuitivos, como também solucionam seus problemas por meio de ensaio e erro, e ainda, procura ajuda em outras pessoas para obter mais informações. Ressalta-se que as pessoas que possuem um alto componente acomodador, tendem a fazer uso da sua força em mudanças triviais, o que pode ocasionar em equívocos ou até mesmo em um fracasso (KOLB; FRY, 1975; CERQUEIRA, 2000).

Estilo Assimilador: combina as etapas de aprendizagem da Conceituação Abstrata (CA) e da Observação Reflexiva (OR). Nesse estilo as pessoas acabam se destacando por seu raciocínio indutivo, como também por meio de sua habilidade para desenvolver modelos abstratos e teóricos. O interesse é maior pelo aspecto lógico do que pelo seu valo prático. Porém um, um forte componente assimilador induz a pessoa para ser incapaz de aplicar seus conhecimentos em situações práticas (KOLB; FRY, 1975; CERQUEIRA, 2000).

Estilo Divergente: apresenta a combinação das etapas de aprendizagem da Experiência Concreta (EC) e da Observação Reflexiva (OR). O estilo divergente aponta que as pessoas usam suas habilidades para combinar as diversas situações sob diferentes ângulos e organizar relações em um todo significativo; possuem como característica atuar de forma eficiente nas situações que exigem ideias novas. São também considerados como criativos, geradores de alternativas, como também tem a capacidade de reconhecerem os problemas e compreender as outras pessoas (KOLB; FRY, 1975; CERQUEIRA, 2000). 
Estilo Convergente: relaciona as etapas de aprendizagem da Conceituação Abstrata (CA) e da Experimentação Ativa (EA). Pelo estilo convergente, o aluno tem mais êxito quando lida com fatos que têm uma única solução correta. Nesse caso, usa o raciocínio hipotético-dedutivo, sendo que o ponto forte está na aplicação prática das ideias, como também na definição do problema e tomada de decisões (KOLB; FRY, 1975; CERQUEIRA, 2000).

Cerqueira (2000, p.53) aponta que a partir da teoria de aprendizagem de Kolb, o estilo de aprendizagem é definido como "um estado duradouro e estável que deriva de configurações consistentes das transações entre o indivíduo e o seu meio ambiente".

\subsection{ESTUDOS ANTERIORES}

Pesquisas tem sido realizadas no sentido de verificar e discutir o Inventário do Estilo de Aprendizagem de David Kolb, como Tirados (1985), Cerqueira (2000), Nogueira et al. (2012), Reis et al. (2012), Lima Filho, Bezerra e Silva (2016), entre outros.

Em seu estudo, Tirados (1985) analisou a influência da natureza dos estudos universitários nos estilos de aprendizagem dos estudantes espanhóis. Para o estudo foi aplicado o Inventário de Kolb (1975), com o propósito de estudar a idoneidade dos termos, validação linguística ou de definição que compõem o inventário. A pesquisa contou com 400 estudantes dos últimos anos de vários cursos universitários, sendo 274 homens e 126 mulheres, na faixa etária de 18 a 29 anos. Tirados constatou que o inventário de Kolb tem validade adequada.

Cerqueira (2000) em sua tese buscou validar o Inventario de Kolb. Aplicou o estudo em 2.552 acadêmicos universitários brasileiros de vários estados de cinco regiões, para verificar se tinha predominância de algum estilo de aprendizagem preferencial. Os acadêmicos pesquisados foram classificados por área do conhecimento, tendo caracterizado oito áreas distintas. O instrumento que foi validado a partir da concordância elevada de 30 juízes/psicólogos revelou o predomínio do estilo de aprendizagem assimilador em todas as áreas do conhecimento.

Nogueira et al. (2012) aplicaram uma pesquisa com o objetivo de verificar se o desempenho dos alunos de educação a distância nas disciplinas de Contabilidade Geral, Gerencial e no módulo de Contabilidade é diferente de acordo com seu estilo de aprendizagem. A pesquisa que foi realizada com 109 alunos de um curso de educação a distância evidenciou que a maior parte dos alunos é do estilo Assimilador (44\%) e Divergente 
(34\%), como também destacaram que não foi possível constatar que os estilos de aprendizagem proporcionassem diferenças no desempenho dos alunos. Os autores ressaltam que o pequeno número de observações, as conclusões não devem ser generalizadas, fícando restrita a população pesquisada.

No estudo de Reis et al. (2012) teve como objetivo identificar os estilos de aprendizagem dos alunos do curso de graduação de Ciências Contábeis. O trabalho que aplicou o modelo de David Kolb, concluiu que o estilo de aprendizagem predominante é o estilo Convergente, sendo o estilo de aprendizagem menos presente o estilo Divergente. Como foram 12 turmas pesquisadas, uma apresentou como estilo de aprendizagem presente na maioria dos alunos o estilo Acomodador. Os autores concluíram que não se devem adotar as mesmas técnicas de aprendizagem para todas as turmas e para todos os alunos, com a expectativa de se ter o mesmo resultado, pois deve ser levado em conta que os estilos de aprendizagem dos acadêmicos diferem entre si, e que apresentam características próprias.

Lima Filho, Bezerra e Silva (2016) realizaram um estudo com o propósito de identificar o estilo de aprendizagem predominante em alunos do Curso de Graduação em Ciências Contábeis nas modalidades de ensino presencial e EAD em instituições públicas e privadas do Estado da Bahia. Com a participação de 459 alunos do curso de Ciências Contábeis e por meio do Learning Style Inventory (LSI), os achados mostraram haver predominância do estilo de aprendizagem assimilador.

Os estudos supracitados e outros desenvolvidos com base em abordagens similares (SILVA; OLIVEIRA NETO, 2010; OLIVEIRA et al. 2013; SILVA JUNIOR; FONTENELE; DA SILVA; 2013; SILVA; ALMEIDA, 2014) acentuam a importância e a preocupação sobre a necessidade de refletir sobre os efeitos metodológicos, psicológicos, como também físicos nos quais é desenvolvido o processo de aprendizagem. Dessa forma, a presente proposta de pesquisa torna-se oportuna por investigar os fatores associativos dos estilos de aprendizagem dos acadêmicos do curso de ciências contábeis da FURB.

\section{PROCEDIMENTOS METODOLÓGICOS}

$\mathrm{Na}$ aplicação prática da presente pesquisa, torna-se necessário selecionar um planejamento específico para usar. Existem vários modelos diferentes, mas nenhum sistema único define todas as variações que devem ser consideradas. Com base em Cooper e Schindler (2003), o presente estudo envolve procedimentos de interrogação/comunicação, por meio de 
questionário. Trata-se de um estudo ex post facto, de rotina real, transversal, estatístico e em condições de campo. Com relação ao objetivo do estudo, é uma pesquisa descritiva, conforme

Quadro 1 Classificação da Pesquisa

\begin{tabular}{|l|l|}
\hline \multicolumn{1}{|c|}{ CATEGORIA } & \multicolumn{1}{c|}{ OPÇÕES } \\
\hline O grau em que as questões de pesquisa foram cristalizadas & Estudo formal \\
\hline O método de coleta de dados & Interrogação/comunicação \\
\hline $\begin{array}{l}\text { O poder do pesquisador de produzir efeitos nas variáveis que } \\
\text { estão sendo estudadas }\end{array}$ & Ex post facto \\
\hline O objetivo do estudo & Descritivo \\
\hline A dimensão de tempo & Transversal \\
\hline O escopo do tópico - amplitude e profundidade - do estudo & Estudo estatístico \\
\hline O ambiente de pesquisa & Ambiente de campo \\
\hline
\end{tabular}

Fonte: Cooper e Schindler (2003. p.129)

Caracteriza-se como "estudo formal", pois é iniciado com uma questão de pesquisa e envolve procedimentos precisos e especificação de fontes de dados. Quanto ao método da coleta de dados, trata-se de uma "interrogação" direta das pessoas cujo comportamento se deseja conhecer. O estudo "ex post facto" se aplica aos investigadores que não tem controle sobre as variáveis no sentido de poderem manipulá-las, é relatado apenas o que aconteceu ou o que esta acontecendo. Como "descritiva", a pesquisa tendo como objetivo principal evidenciar as características de determinadas populações ou fenômenos. É “transversal”, pois os resultados são agrupados uma vez e representam um instantâneo de um determinado momento. Sobre os "estudos estatísticos", justifica-se, pois são voltados para a amplitude e não para a profundidade, onde se busca captar as características de uma população ao fazer inferências das características de uma amostra, em um "ambiente de campo" (COOPER; SCHINDLER, 2003).

Para a realização da pesquisa, foi utilizado o questionário fechado, dividido em 2 partes e contendo no total 7 perguntas. Quanto às perguntas abordadas nos questionários, segundo Cooper e Schindler (2003, p. 278), “as decisões de estratégia de resposta (o tipo de pergunta usada) dependem do conteúdo e dos objetivos das perguntas específicas".

A pesquisa foi realizada nos meses de maio e junho de 2015, que constituiu-se primeiramente por 166 acadêmicos dos 271 matriculados e com frequência no Curso de Graduação em Ciências Contábeis da Universidade Regional de Blumenau (FURB), Brasil. Do total de 166 pesquisados, 15 questionários foram descartados da análise por não terem sido preenchidos corretamente. Dessa forma, foram validados 151 questionários. As questões 
de 1 a 6 traçam o perfil dos respondentes quanto a: gênero, idade, semestre no curso, área de atuação profissional, tipo de empresa em que atua, tempo de dedicação aos estudos por semana.

Já a questão 7 que foi subdividida em 12 sentenças no inventário de estilo de aprendizagem, tem como propósito identificar o estilo de aprendizagem predominante nos acadêmicos. O Inventário de Estilo de Aprendizagem (KOLB, 1975) evidencia a maneira pela qual o aluno aprende e como lida com as ideias e as situações do dia-a-dia de sua vida. $\mathrm{O}$ inventário contém 12 sentenças, sendo que cada uma tem quatro terminações (A, B, C, D). O aluno classificou as terminações de cada sentença de forma a retratar a maneira como atua ao ter que aprender algo. Com base no exposto, fazendo uso do espaço disponível, o acadêmico teve que classificar com "4" a terminação da sentença que descreve a sentença como aprende melhor descendo até chegar a "1" para a terminação da sentença que considera que é a maneira menos provável em que aprenderia algo.

Para o tratamento estatístico, foi adotada a análise descritiva dos dados, na sequência, foi aplicado a análise de correspondências múltiplas (ANACOR), com a utilização do software LHStat ${ }^{\circledR}$. Essa técnica se concentra na verificação da associação entre variáveis que podem ser categóricas e que geram tabelas de contingência (FÁVERO et al. 2009). Em situações de trabalhos em que o número de variáveis e categorias é menor, torna-se necessário usar o teste Qui-quadrado para avaliar se a informação nas linhas das tabelas é independente ou não das contidas nas colunas. Ao contrário, quando ocorre de o conjunto de dados ser maior, como é o caso do presente estudo, o uso da técnica Qui-quadrado apresenta limitações, diante da demanda de relacionar um maior número de variáveis e categorias. Portanto, nestes casos uma opção é a análise de correspondências (ANACOR).

Segundo Fávero et al. (2009), a análise de correspondência (ANACOR) e a análise de homogeneidade (HOMALS) são técnicas de interdependência que buscam estudar a relação entre variáveis qualitativas, e que possibilita ao pesquisador a visualização de associações, por meio de mapas perceptuais que oferecem a noção de proximidade. Dessa forma, exibe-se as associações entre o conjunto de variáveis categóricas em um mapa ou gráfico perceptual, o que possibilita um exame visual de qualquer padrão ou estrutura de dados.

Com base no exposto, a análise de correspondências múltiplas permite estudar associações entre variáveis qualitativas, e a natureza do agrupamento de dados deste artigo conduz a resultados relevantes com a observação sob o uso deste método. 


\section{ANÁLISE E INTERPRETAÇÃO DOS DADOS}

Neste tópico são apresentados os estilos de aprendizagem dos acadêmicos pesquisados e suas características, como também a aplicação do teste de análise de correspondência para verificar os fatores de associação com os estilos de aprendizagem.

\subsection{OS ESTILOS DE APRENDIZAGEM}

A seguir apresentam-se os resultados do questionário aplicado, na forma de tabelas e com observações referentes à interpretação e análise dos dados. De acordo com a Tabela 1, $48,3 \%$ (73) dos pesquisados tem o estilo de aprendizagem convergente, 33,1\% (50) o assimilador, 11,3\% (17) o divergente e 7,3\% (11) o acomodador. A predominância do estilo convergente na pesquisa corrobora com o resultado de Reis et al. (2012). Lima Filho, Bezerra e Silva (2016) argumentam que o docente da área de contabilidade precisar estar consciente das exigências do mercado de trabalho e das diferenças dos estilos de aprendizagem dos seus acadêmicos para o planejamento e aplicação da metodologia de ensino elaborada.

Tabela 1 Estilo de Aprendizagem

\begin{tabular}{c|c|c|c|c|c}
\hline & Acomodador & Assimilador & Convergente & Divergente & TOTAL \\
\hline Frequência & 11 & 50 & 73 & 17 & 151 \\
Percentual (\%) & $7,3 \%$ & $33,1 \%$ & $48,3 \%$ & $11,3 \%$ & $100,0 \%$ \\
\hline
\end{tabular}

Fonte: Dados da Pesquisa (2015).

Identificado os estilos de aprendizagem, a Tabela 2 mostra o estilo de aprendizagem por gênero. Dos pesquisados, 60,9 \% (92) são do gênero feminino e 39,1\% (59) masculino, sendo que o estilo de aprendizagem convergente predomina nos dois gêneros, com destaque para o feminino.

Tabela 2 Estilo de Aprendizagem e Gênero

\begin{tabular}{c|c|c|c|c|c}
\hline & Acomodador & Assimilador & Convergente & Divergente & TOTAL \\
\hline Feminino & $9(6,0 \%)$ & $27(17,9 \%)$ & $41(27,2 \%)$ & $15(9,9 \%)$ & $92(60,9 \%)$ \\
Masculino & $2(1,3 \%)$ & $23(15,2 \%)$ & $32(21,2 \%)$ & $2(1,3 \%)$ & $59(39,1 \%)$ \\
\hline TOTAL & $11(7,3 \%)$ & $50(33,1 \%)$ & $73(48,3 \%)$ & $17(11,3 \%)$ & $151(100,0 \%)$ \\
\hline
\end{tabular}

Fonte: Dados da Pesquisa (2015).

No que diz respeito à faixa etária, a Tabela 3 demonstra que o estilo de aprendizagem convergente é predominante em todas as faixas etárias. Porém, vale destacar o percentual 
relevante do estilo de aprendizagem assimilador nas faixas etárias de até 20 anos com 14,6\% (22) e de 13,9\% (21) na faixa etária de 21 a 25 anos. A pesquisa de Cerqueira (2000), que foi a única encontrada que evidenciou os resultados por faixa etária dos universitários, o estilo assimilador predominou em todas as faixas etárias.

Tabela 3 Estilo de Aprendizagem e Idade

\begin{tabular}{c|c|c|c|c|c}
\hline & Acomodador & Assimilador & Convergente & Divergente & TOTAL \\
\hline Até 20 anos & $5(3,3 \%)$ & $22(14,6 \%)$ & $36(23,8 \%)$ & $11(7,3 \%)$ & $74(49,0 \%)$ \\
De 21 a 25 anos & $3(2,0 \%)$ & $21(13,9 \%)$ & $26(17,2 \%)$ & $2(1,3 \%)$ & $52(34,4 \%)$ \\
De 26 a 30 anos & $2(1,3 \%)$ & $4(2,6 \%)$ & $7(4,6 \%)$ & $3(2,0 \%)$ & $16(10,6 \%)$ \\
Acima de 30 anos & $1(0,7 \%)$ & $3(2,0 \%)$ & $4(2,6 \%)$ & $1(0,7 \%)$ & $9(6,0 \%)$ \\
\hline TOTAL & $11(7,3 \%)$ & $50(33,1 \%)$ & $73(48,3 \%)$ & $17(11,3 \%)$ & $151(100,0 \%)$ \\
\hline
\end{tabular}

Fonte: Dados da Pesquisa (2015).

A Tabela 4 compara o estilo de aprendizagem do aluno por semestre.

Tabela 4 Estilo de Aprendizagem e Semestre

\begin{tabular}{c|c|c|c|c|c}
\hline & Acomodador & Assimilador & Convergente & Divergente & TOTAL \\
\hline $\mathbf{1}^{\mathbf{o}}$ SEM & $4(2,6 \%)$ & $1(0,7 \%)$ & $5(3,3 \%)$ & $9(6,0 \%)$ & $19(12,6 \%)$ \\
$\mathbf{2}^{\mathbf{o}}$ SEM & $1(0,7 \%)$ & $6(4,0 \%)$ & $7(4,6 \%)$ & $4(2,6 \%)$ & $18(11,9 \%)$ \\
$\mathbf{3}^{\mathbf{o}}$ SEM & $2(1,3 \%)$ & $13(8,6 \%)$ & $16(10,6 \%)$ & $0(0,0 \%)$ & $31(20,5 \%)$ \\
$\mathbf{4}^{\mathbf{o}}$ SEM & $1(0,7 \%)$ & $5(3,3 \%)$ & $8(5,3 \%)$ & $1(0,7 \%)$ & $15(9,9 \%)$ \\
$\mathbf{5}^{\mathbf{o}}$ SEM & $1(0,7 \%)$ & $10(6,6 \%)$ & $13(8,6 \%)$ & $2(1,3 \%)$ & $26(17,2 \%)$ \\
$\mathbf{6}^{\mathbf{o}}$ SEM & $2(1,3 \%)$ & $4(2,6 \%)$ & $8(5,3 \%)$ & $1(0,7 \%)$ & $15(9,9 \%)$ \\
$\mathbf{7}^{\mathbf{o}}$ SEM & $0(0,0 \%)$ & $6(4,0 \%)$ & $8(5,3 \%)$ & $0(0,0 \%)$ & $14(9,3 \%)$ \\
$\mathbf{8}^{\mathbf{o}}$ SEM & $0(0,0 \%)$ & $5(3,3 \%)$ & $8(5,3 \%)$ & $0(0,0 \%)$ & $13(8,6 \%)$ \\
\hline TOTAL & $11(7,3 \%)$ & $50(33,1 \%)$ & $73(48,3 \%)$ & $17(11,3 \%)$ & $151(100,0 \%)$ \\
\hline
\end{tabular}

Fonte: Dados da Pesquisa(2015).

Nota-se na Tabela 4 que o estilo que mais predomina na maioria dos semestres é o convergente. É importante frisar que o estilo de aprendizagem divergente tem maior representatividade entre os alunos do $1^{\mathrm{o}}$ semestre, com 6\% (9). No estudo de Cerqueira (2000), que foi o único encontrado que apresentou os resultados por semestre de atuação dos universitários, o estilo assimilador predominou em todos os semestres. 
Tabela 5 Estilo de Aprendizagem e Empresa de Atuação

\begin{tabular}{c|c|c|c|c|c}
\hline & Acomodador & Assimilador & Convergente & Divergente & TOTAL \\
\hline Privada & $10(6,6 \%)$ & $44(29,1 \%)$ & $66(43,7 \%)$ & $12(7,9 \%)$ & $132(87,4 \%)$ \\
Pública & $0(0,0 \%)$ & $2(1,3 \%)$ & $4(2,6 \%)$ & $2(1,3 \%)$ & $8(5,3 \%)$ \\
Apenas Estuda & $1(0,7 \%)$ & $4(2,6 \%)$ & $3(2,0 \%)$ & $3(2,0 \%)$ & $11(7,3 \%)$ \\
\hline TOTAL & $11(7,3 \%)$ & $50(33,1 \%)$ & $73(48,3 \%)$ & $17(11,3 \%)$ & $151(100,0 \%)$ \\
\hline
\end{tabular}

Fonte: Dados da Pesquisa (2015).

Ao comparar o estilo de aprendizagem com o tipo de empresa de atuação, os achados da Tabela 5 mostram que o estilo convergente se destaca nos acadêmicos que atuam em empresas privadas e públicas, porém, entre os que apenas estudam, prevaleceu o estilo assimilador com 2,6\% (4).

Tabela 6 Estilo de Aprendizagem e Área de Atuação

\begin{tabular}{c|c|c|c|c|c}
\hline & Acomodador & Assimilador & Convergente & Divergente & TOTAL \\
\hline Contabilidade & $5(3,3 \%)$ & $25(16,6 \%)$ & $42(27,8 \%)$ & $7(4,6 \%)$ & $79(52,3 \%)$ \\
Outra Área & $5(3,3 \%)$ & $21(13,9 \%)$ & $28(18,5 \%)$ & $7(4,6 \%)$ & $61(40,4 \%)$ \\
Apenas Estuda & $1(0,7 \%)$ & $4(2,6 \%)$ & $3(2,0 \%)$ & $3(2,0 \%)$ & $11(7,3 \%)$ \\
\hline TOTAL & $11(7,3 \%)$ & $50(33,1 \%)$ & $73(48,3 \%)$ & $17(11,3 \%)$ & $151(100,0 \%)$ \\
\hline
\end{tabular}

Fonte: Dados da Pesquisa (2015).

Quando questionados sobre a área de atuação, similar a tabela anterior, a Tabela 6 revela que a maioria dos acadêmicos que atuam na área de contabilidade, como também em outra área, possuem o estilo convergente. Já os que apenas estudam, predomina o estilo assimilador. Vale destacar que 52,3\% (79) dos pesquisados já atuam na área de contabilidade.

Os alunos que dedicam maior tempo aos estudos em casa ou na biblioteca por semana, na maioria tem o estilo de aprendizagem convergente, conforme a Tabela 7. O estilo assimilador é predominante apenas entre os alunos que se dedicam de 2 a 4 horas aos estudos por semana, com 19,9\% (30). Do total de pesquisados, 50,3\% (76) se dedicam aos estudos de 2 a 4 horas por semana. 
Tabela 7 Estilo de Aprendizagem e Horas de Estudo por Semana

\begin{tabular}{c|c|c|c|c|c}
\hline & Acomodador & Assimilador & Convergente & Divergente & TOTAL \\
\hline Até 1 hora & $4(2,6 \%)$ & $11(7,3 \%)$ & $28(18,5 \%)$ & $3(2,0 \%)$ & $46(30,5 \%)$ \\
De 2 a 4 horas & $6(4,0 \%)$ & $30(19,9 \%)$ & $29(19,2 \%)$ & $11(7,3 \%)$ & $76(50,3 \%)$ \\
De 5 a 7 horas & $1(0,7 \%)$ & $7(4,6 \%)$ & $11(7,3 \%)$ & $1(0,7 \%)$ & $20(13,2 \%)$ \\
De 8 a 10 horas & $0(0,0 \%)$ & $1(0,7 \%)$ & $2(1,3 \%)$ & $0(0,0 \%)$ & $3(2,0 \%)$ \\
Acima de 10 & $0(0,0 \%)$ & $1(0,7 \%)$ & $3(2,0 \%)$ & $2(1,3 \%)$ & $6(4,0 \%)$ \\
\hline horas & $11(7,3 \%)$ & $50(33,1 \%)$ & $73(48,3 \%)$ & $17(11,3 \%)$ & $151(100,0 \%)$ \\
\hline TOTAL & &
\end{tabular}

Fonte: Dados da Pesquisa (2015).

Com base nos resultados acima, constata-se a predominância do estilo de aprendizagem "convergente" nos acadêmicos pesquisados e em algumas situações específicas o estilo "assimilador".

\subsection{ANÁLISE DE CORRESPONDÊNCIAS MÚLTIPLAS (ANACOR)}

Com a utilização do software LHStat ${ }^{\circledR}$, foi realizada a análise de correspondências múltiplas, sobre variáveis categóricas, das questões em conjunto para realização de correlação, conforme mapa perceptual que segue abaixo, após a identificação dos fatores para análise.

A distribuição dos pontos possibilita a análise graficamente da associação dos estilos de aprendizagem dos acadêmicos com os seguintes fatores: gênero, idade, semestre matriculado no curso, área de atuação profissional, tipo de empresa em que atua e quantidade de horas de dedicação aos estudos por semana. Os 151 respondentes estão representados no mapa perceptual por meio das variáveis.

O mapa de percepções do Gráfico 1 está subdividido em quatro quadrantes. No primeiro quadrante, parte superior lado direito, constata-se que os fatores apresentados estão dispersos, ou seja, as variáveis desse quadrante apresentam-se dissimilares entre os grupos, com exceção das variáveis tipo de empresa e área de atuação. No caso dessas duas variáveis, compreendem os alunos que apenas estudam.

Quanto mais afastados do centróide e mais próximos estiverem às variáveis, maior é poder associativo. No segundo quadrante, parte superior lado esquerdo, apesar de algumas variáveis estarem na região do centróide, percebe-se a formação de grupo. Com a presença dos estilos de aprendizagem assimilador e convergente, nota-se que há associação com acadêmicos do gênero masculino, com idade entre 21 a 25 anos, que estão cursando o $6^{\circ}, 7^{\circ} \mathrm{e}$ 
$8^{\circ}$ semestre, que se dedicam aos estudos de 8 a 10 horas por semana, e ainda, que já atuam na área de contabilidade.

Gráfico 1 Mapa Perceptual dos Fatores Associativos dos Estilos de Aprendizagem

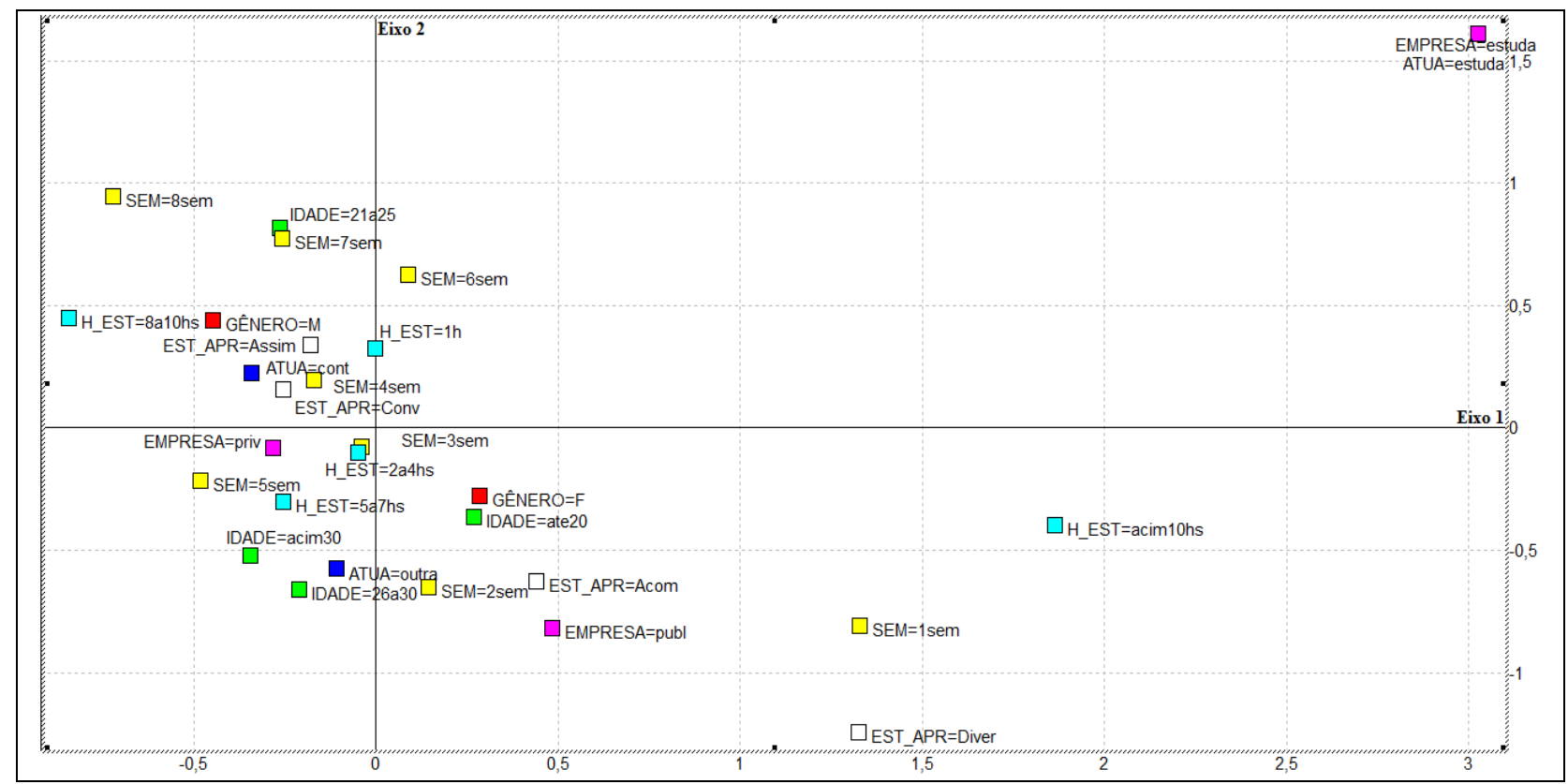

Fonte: Dados da Pesquisa, Software LHStat ${ }^{\circledR}(2015)$

Legenda: $\left(E S T \_A P R=A c o m\right)=$ estilo de aprendizagem acomodador; $\left(E S T \_A P R=A s i m\right)=$ estilo de aprendizagem assimilador; $\left(\overline{E S T} \_\mathrm{APR}=\mathrm{Conv}\right)=$ estilo de aprendizagem convergente; $\left(\mathrm{EST} \_\mathrm{APR}=\mathrm{Diver}\right)=$ estilo de aprendizagem divergente; $(\mathrm{GÊNERO}=\mathrm{F})=$ gênero feminino; $(\mathrm{GÊNERO}=\overline{\mathrm{M}})=$ gênero masculino; $(\mathrm{IDADE}=$ ate 20$)=$ até 20 anos de idade; $(\mathrm{IDADE}=21 \mathrm{a} 25)=$ de 21 a 25 anos de idade; $($ IDADE $=26 \mathrm{a} 30)=$ de 26 a 30 anos de idade; $\left(\right.$ IDADE=acim30) $=$ acima de 30 anos de idade; $(\mathrm{SEM}=1 \mathrm{sem})=$ matriculado no $1^{\circ}$ semestre; $(\mathrm{SEM}=2 \mathrm{sem})=$ matriculado no $2^{\circ}$ semestre; $(\mathrm{SEM}=3 \mathrm{sem})=$ matriculado no $3^{\circ}$ semestre; $(\mathrm{SEM}=4 \mathrm{sem})=$ matriculado no $4^{\circ}$ semestre; $(\mathrm{SEM}=5 \mathrm{sem})=$ matriculado no $5^{\circ}$ semestre; $(\mathrm{SEM}=6 \mathrm{sem})=$ matriculado no $6^{\circ}$ semestre; $(\mathrm{SEM}=7 \mathrm{sem})=$ matriculado no $7^{\circ}$ semestre; $\quad(\mathrm{SEM}=8 \mathrm{sem})=$ matriculado no $8^{\circ}$ semestre; $(\mathrm{ATUA}=$ cont $)=$ atuação na área contábil; $(\mathrm{ATUA}=$ outra $)=$ atuação em outra área; $(\mathrm{ATUA}=$ estuda $)=$ apenas estuda; $($ EMPRESA $=$ publ $)=$ atuação em empresa pública; $($ EMPRESA $=$ priv $)=$ atuação em empresa privada; $($ EMPRESA $=$ estuda $)=$ apenas estuda; $\left(H_{-}\right.$EST $\left.=1 \mathrm{~h}\right)=1$ hora de estudo por semana; $\left(H_{-} E S T=2 \mathrm{a} 4 \mathrm{hs}\right)=$ de 2 a 4 horas de estudo por semana; (H_EST $=5 \mathrm{a} \overline{\mathrm{h} h})=$ de 5 a 7 horas de estudo por semana; $\left(\overline{\mathrm{H}} \_\mathrm{EST}=8 \mathrm{a} 10 \mathrm{hs}\right)=$ de $8 \mathrm{a}$ 10 horas de estudo por semana; (H_EST=acim10hs) $=$ acima de 10 horas de estudo por semana.

Os resultados do segundo quadrante revelam que os acadêmicos têm uma boa dedicação de horas aos estudos por semana, de 8 a 10 horas. Essa quantidade de horas de estudo pode estar relacionado também ao fato de estarem no período de desenvolvimento do trabalho de conclusão de curso (TCC), modalidade de atividade que exige mais horas de dedicação. A associação das variáveis apresentadas com os estilos de aprendizagem assimilador e convergente, mostram que os alunos consideram quanto ao primeiro estilo, que é mais importante que uma teoria tenha um sentido lógico do que um valor prático (BARROS, 2011). Os alunos se destacam quando se trata de entender uma ampla gama de 
informações e dar-lhe uma forma concisa e lógica. Já o estilo convergente revela que os acadêmicos se destacam quando se trata de encontrar o uso prático das ideias e teorias, prefere manejar situações ou problemas técnicos, a temas sociais e interpessoais, como também têm a capacidade de resolver problemas e tomar decisões que se baseiam em encontrar soluções para questões ou problemas (CERQUEIRA, 2000).

No terceiro quadrante, parte inferior lado esquerdo, percebe-se que não teve um estilo de aprendizagem que apresentasse uma forte associação com as variáveis estudadas. Porém, consta nesse quadrante alguns fatores que tem certa associação, tais como: acadêmicos do $3^{\circ}$ semestre que se dedicam aos estudos de 2 a 4 horas por semana e do $5^{\circ}$ semestre que se dedicam aos estudos de 5 a 7 horas por semana. É constatado também a associação com a idade que varia de 26 a 30 anos, como também acima de 30 anos.

Já no quarto quadrante, parte inferior lado direito, é evidenciado que o estilo de aprendizagem acomodador tem uma maior associação com as variáveis do quadrante, porém, deve ser destacado que o estilo de aprendizagem divergente também exerce influência, mas com menor intensidade. Portanto, os dois estilos de aprendizagem têm uma associação com os acadêmicos do gênero feminino, com idade de até 20 anos, que estão cursando o $1^{\circ}$ e $2^{\circ}$ semestre, que se dedicam aos estudos acima de 10 horas por semana, como também, que atuam em empresa pública.

Os achados do quarto quadrante revelam que os acadêmicos calouros são os que mais se dedicam aos estudos por semana, acima de 10 horas. Pelo fato dos alunos terem ingressado recentemente ao curso e devido a existência de muitas novidades informações (conteúdo) da área específica, torna-se um fator que pode influenciar na alta quantidade de horas de dedicação aos estudos. A associação das variáveis apresentadas com os estilos de aprendizagem acomodador e divergente destaca que os alunos desse grupo, especificamente quanto ao primeiro estilo, no momento de resolver um problema, pode ser que confie mais nas pessoas para conseguir informações do que em sua própria análise (BARROS, 2011). Já quanto ao estilo divergente, os acadêmicos atuam melhor quando se trata de observar situações concretas de diferentes pontos de vista, e sua maneira de enfrentar as situações consiste mais em observar do que atuar. Atuam bem também nas situações que pedem novas ideias, são criativos, geradores de alternativas, compreendem as pessoas e reconhecem os problemas (CERQUEIRA, 2000). 
Com base no exposto, revela-se achados importantes, como a associação dos estilos de aprendizagem acomodador e divergente com os acadêmicos da fase inicial do curso, com o gênero feminino, com acadêmicos de idade até 20 anos e com os acadêmicos que tem uma alta dedicação de horas aos estudos por semana. Observa-se também a associação dos estilos assimilador e convergente com os acadêmicos da fase final do curso, do gênero masculino, com idade entre 21 a 25 anos e que também tem uma carga horária relevante de horas de dedicação aos estudos por semana.

Portanto, a análise de correspondência, técnica multivariada exploratória possibilitou mostrar as associações em um espaço multidimensional, o que permitiu representar graficamente a natureza das relações existentes.

\section{CONSIDERAÇÕES FINAIS E RECOMENDAÇÕES}

Como proposto neste estudo, o objetivo geral consistiu em evidenciar os fatores associativos dos estilos de aprendizagem dos acadêmicos do curso de ciências contábeis da FURB. Em busca de uma resposta para a questão principal desta pesquisa, foi utilizado um questionário com sete questões consideradas essenciais para o objetivo da pesquisa.

Com base nos resultados alcançados e analisados por meio do questionário, foi possível identificar o estilo de aprendizagem predominante dos alunos do curso. Foi constatado que 48,3\% (73) dos pesquisados que tiveram os questionários validados, possuem o estilo de aprendizagem convergente, ou seja, possuem um estilo de aprendizagem que revela que os acadêmicos acabam se destacando encontram o uso prático das ideias e teorias, eles preferem situações ou problemas técnicos, a temas sociais e interpessoais. Ainda foi constatado que $33,1 \%$ (50) possuem o estilo assimilador. Os estilos acomodador e divergente somados foram identificados em 18,6\% (28) dos acadêmicos.

Com a utilização da análise de correspondências múltiplas por meio do software LHStat ${ }^{\circledR}$, foi realizada a análise de correspondências da variável categórica "estilo de aprendizagem" com as variáveis gênero, idade, semestre atual no curso, área de atuação profissional, tipo de empresa em que atua e quantidade de horas de dedicação aos estudos por semana. Com o mapa perceptual, o estudo revelou a associação dos estilos de aprendizagem acomodador e divergente com os acadêmicos da fase inicial do curso, com o gênero feminino, com acadêmicos de idade até 20 anos e com os acadêmicos que tem uma alta dedicação de horas aos estudos por semana. Os achados também revelaram a associação dos estilos 
assimilador e convergente com os acadêmicos da fase final do curso, do gênero masculino, com idade entre 21 a 25 anos e que também tem uma carga horária relevante de horas de dedicação aos estudos por semana.

Como base no apresentado, pode concluir que o objetivo da pesquisa foi alcançado, e que tão importante quanto saber o estilo de aprendizagem do acadêmico em Ciências Contábeis é saber explorar o estilo e suas associações para um melhor ensino-aprendizagem. Portanto, o processo de compreensão de como os acadêmicos aprendem e o porquê se diferenciam perante os demais torna-se uma parte de um processo essencial da educação e das inovações, pois o processo educacional pode ser sempre aperfeiçoado. O presente estudo apresenta informações que são úteis na avaliação do processo de aprendizagem pelos docentes e para a identificação dos pontos fortes e fracos dos acadêmicos.

Destaca-se que os resultados obtidos nesta pesquisa limitam-se com alunos do curso de Ciências Contábeis da FURB. Recomenda-se que sejam feitas novas investigações sobre a identificação e comparação dos estilos de aprendizagem dos alunos que cursam Ciências Contábeis em outras regiões. Outra recomendação é que novas investigações relacionem a influência dos estilos de aprendizagem nos desempenhos acadêmicos.

\section{REFERÊNCIAS}

BARROS, D. M. V.. Estilos de aprendizagem na atualidade-volume 1. Lisboa: Educação e Tecnologias, 2011

CERQUEIRA, T. C. S.. Estilos de aprendizagem em universitários. Campinas. Tese de Doutorado em Educação - Faculdade de Educação, Universidade Estadual de Campinas (UNICAMP). Campinas, 2000.

CLAXTON, C.S.; MURRELL, P.H.. Learning Styles: Inplications for Improving Practices ASHE-ERIC Higher Education Report, 4, Washington, 1987.

COOPER, D.R.; SCHINDLER, P. S.. Métodos de pesquisa em administração. (7.ed.). Porto Alegre: Bookman, 2003.

CORDEIRO, R. A.; SILVA, A. B.. Os estilos de aprendizagem influenciam o desempenho acadêmico dos estudantes de finanças? Revista de Administração da UFMS, v.5, n.2, p. 243-261, 2012.

FÁVERO, L. P.; BELFIONE, P.; DA SILVA, F.L.; CHAN, B.L.. Análise de Dados: Modelagem Multivariada para Tomada de Decisões. (1.ed.) Rio de Janeiro: Campos Elsevier, 2009. 
KOLB, D.; FRY, R.. Toward an apllied theory of experiential learning. In: C. COOPER (ed). Theories of Group Process. London: John Wiley, 1975.

KOLB, D. A.. The learning style inventory: technical manual. Boston: Ma.McBer, 1976.

. Experimental learning: experience as the source of learning and

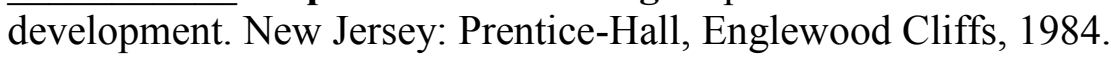

LEITÃO, M.B.P.. Estilos de aprendizagem sob a ótica da psicologia evolucionista.

Dissertação (Mestrado em Psicobiologia) - Departamento de Fisiologia, Universidade Federal do Rio Grande do Norte. Natal, 2006.

LIMA FILHO, R.; BEZERRA, E.; SILVA, T.. Estilo de aprendizagem dos alunos do curso de Ciências Contábeis. Revista Gestão Universitária na América Latina - GUAL, v.9, n.2, p. 95-112, 2016. doi:http://dx.doi.org/10.5007/39567

NOGUEIRA, D.R.; ESPEJO, M.M.S.B.; REIS, L.G.; VOESE, S.B.. Estilos de aprendizagem e desempenho em educação a distância: um estudo empírico com alunos da s disciplinas de contabilidade geral e gerencial. Revista de Educação e Pesquisa em Contabilidade (REPeC), Brasília, v.6, n.1, p. 54-72, 2012.

NOGUEIRA, D.R.; COSTA, J.M.; TAKAMATSU, R. T.; REIS, L.G.. Fatores que impactam o desempenho acadêmico: uma análise com discentes do curso de ciências contábeis no ensino presencial. Revista de Informação Contábil, v.7, n.3, p. 51-62, 2013.

OLIVEIRA, A.J.; RAFFAELLI, S.C.D.; COLAUTO, R.D.; NOVA, S.P.D.C.C.. Estilos de aprendizagem e estratégias ludo pedagógicas: percepções no ensino da contabilidade. Advances in Scientific and Applied Accounting, v.6, n.2, p. 236-262, 2013.

REIS, L.G.; PATON, C.; NOGUEIRA, D.R.. Estilos de aprendizagem: uma análise dos alunos do curso de ciências contábeis pelo método Kolb. Revista Enfoque: Reflexão Contábil, v.31, n.1, p. 53-66, 2012.

SILVA, D.M.; OLIVEIRA NETO, J.D.. O impacto dos estilos de aprendizagem no ensino de contabilidade. Contabilidade Vista \& Revista, v.21, n.4, p.123-156, 2011.

SILVA, R.P.A.; ALMEIDA, S.R.. Estilos de aprendizagem e variáveis influenciadoras: um estudo com entre alunos o curso de ciências contábeis em uma universidade pública. Revista de estilos de aprendizaje, v.7, n. 14, p. 203-228, 2014.

SILVA JUNIOR, C.A.P.; FONTENELE, HB.; DA SILVA, A.N.R.. Estilos de ensino vs. estilos de aprendizagem no processo de ensino-aprendizagem: uma aplicação em Transportes. Transportes, v.21, n.2, p. 30-37, 2013.

TIRADOS, M. R. G.. Influência de la Natureza de los Estudios Universitarios en los Estilos de Aprendizales de los sujeitos. 1985. Tese (Dourado em Psicologia) Faculdade de Psicologia - Universidad Complitense de Madrid, 1985. 